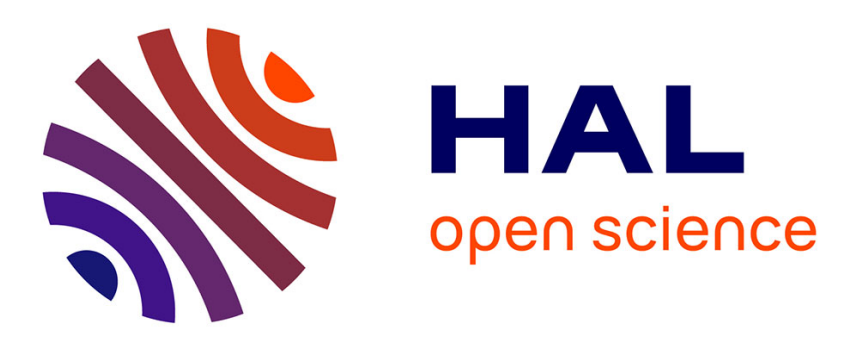

\title{
Les Kanak à l'épreuve de la colonisation. Entre histoire et anthropologie
}

Isabelle Merle

\section{To cite this version:}

Isabelle Merle. Les Kanak à l'épreuve de la colonisation. Entre histoire et anthropologie. Revue d'Histoire Moderne et Contemporaine, 2016, 63 (1), pp.195-204, Vol. 63(1). 10.3917/rhmc.631.0195 SMASH . halshs-01350519

\section{HAL Id: halshs-01350519 https://shs.hal.science/halshs-01350519}

Submitted on 25 Feb 2020

HAL is a multi-disciplinary open access archive for the deposit and dissemination of scientific research documents, whether they are published or not. The documents may come from teaching and research institutions in France or abroad, or from public or private research centers.
L'archive ouverte pluridisciplinaire HAL, est destinée au dépôt et à la diffusion de documents scientifiques de niveau recherche, publiés ou non, émanant des établissements d'enseignement et de recherche français ou étrangers, des laboratoires publics ou privés. 
Revue d'Histoire Moderne \& Contemporaine

63-1, janvier-mars 2016

\section{Rubrique : Lectures}

\section{Titre courant :}

\section{Les Kanak à l'épreuve de la colonisation. Entre histoire et anthropologie}

\section{À propos de :}

Alban Bensa, Kacuè Yvon GoRomoedo, Adrian Muckle, Les sanglots de l'aigle pêcheur. Nouvelle-Calédonie. La guerre kanak de 1917, Toulouse, Éditions Anacharsis, 2015, 716 p., ISBN 978-2-914777-97-1

Michel NAEPEls, Conjurer la guerre. Violence et pouvoir à Houaïlou (Nouvelle-Calédonie), Paris, Éditions de l'EHESS, 2013, 287 p., ISBN 978-2-7132-2376-1

Isabelle MERLE

Nous disposons aujourd'hui de deux nouveaux ouvrages en langue française qui éclairent en profondeur certains segments essentiels de l'histoire de la Nouvelle-Calédonie du point de vue kanak. En 2013, l'anthropologue Michel Naepels (anthropologue, EHESS) publie Conjurer la guerre. Violence et pouvoir à Houaïlou (Nouvelle-Calédonie). En 2015, Alban Bensa (anthropologue, EHESS), Kacué Yvon Goromeodo (professeur de langue Paicî) et Adrian Muckle (historien, University of Wellington), font paraître Les sanglots de l'aigle pêcheur. Nouvelle Calédonie. La guerre kanak de 1917. Ce dernier livre s'appuie, pour la partie historique, sur le travail de thèse publié en 2012 par Adrian Muckle sous le titre Specters of Violence in a Colonial Context. New Caledonia, 1917 aux presses universitaires d'Hawaii.

\section{La guerre comme principe d'action et de récit}

Les deux ouvrages, ici présentés ${ }^{1}$, renouvellent considérablement 1'historiographie de la

\footnotetext{
${ }^{1}$ Je remercie Monika Stern pour la relecture attentive qu'elle a faite de ce texte.
} 
Nouvelle-Calédonie sous plusieurs angles. Ils opèrent tout d'abord une véritable percée dans l'épaisseur de l'histoire kanak jusque-là trop imparfaitement décryptée en plaçant résolument au cœur de leur effort d'analyse, des acteurs et des groupes qu'ils cherchent à historiciser rigoureusement ainsi que leurs logiques d'action, interprétations et horizons d'attente dans le contexte qui est le leur d'un monde profondément bouleversé par l'intrusion des Européens et l'emprise progressive et oppressante de la colonisation. Ces deux ouvrages partagent ensuite une même méthodologie dont le trait commun serait l'attention extrêmement précise portée à la question des sources sur lesquels ils s'appuient, orales ou écrites, soigneusement analysées à l'aune du contexte de leur fabrication ou énonciation qu'il s'agisse d'entretiens recueillis auprès d'interlocuteurs nommés et situés, qu'il s'agisse des récits ou poésies longuement narrés devant l'ethnologue ou le linguiste et les versions du passé qu'elles sous tendent, qu'il s'agisse des archives dites coloniales lues dans le fil ou le contrefil du texte avec une attention toute particulière portée à la voix des subalternes qui parfois émerge ou à l'inverse reste inaccessible étouffée par le filtre d'observateurs européens aveugles aux situations dans lesquels ils sont plongés. Dans ce corpus des archives dites coloniales, on trouve aussi les premiers écrits kanak, plus nombreux qu'on ne le croit habituellement, rédigés dès la fin du XIXe siècle sous l'impulsion des églises et des circonstances témoignant d'un apprentissage précoce de l'écriture dans ces sociétés mélanésiennes d'oralité et des enjeux concrets et divers de dynamiques de mise en récit pour fixer, protéger, expliquer, les fondements d'univers sociaux et culturels alors violemment menacés. Nous insistons sur cette attention portée aux sources - récits, paroles, écrits - que partagent ces auteurs, historiens, anthropologues ou linguistes, par delà les frontières de leurs disciplines respectives, qui se situe au cœur de l'enquête résolument inscrite dans le temps, l'histoire et les enjeux de mémoire. Ces deux ouvrages partagent enfin une ligne d'attention commune : la guerre. Celle-ci constitue l'événement central autour duquel se sont concentrés Alban Bensa, Yvon Goromoedo et Adrian Muckle, cette guerre de 1917 qui fut longtemps appelée «insurrection ou révolte » selon une terminologie toute coloniale. Hisser l'événement à la dignité de la guerre, c'est rendre justice au sens donné par les acteurs kanaks qui se levèrent pour combattre avec l'ultime espoir de «jeter les Blancs à la mer ${ }^{2}$ s'appuyant sur leur expérience de guerres et de pratiques anciennes - monnaies de guerre, pierre propitiatoires,

\footnotetext{
${ }^{2}$ Formule utilisée dans plusieurs récits kanak. Pour exemple cf. Bensa, Goromoedo, Muckle, Les sanglots de l'aigle pêcheur, p. 79.
} 
paniers sacrés, appel à la protection des ancêtres ou aux alliances claniques, tout autant que les expériences nouvelles acquises aux contacts des européens, l'usage du fusil, l'utilisation des chevaux, l'intensité du travail pour élargir le front des alliances rendu nécessaire par l'asymétrie des forces, l'effort pour évaluer la parole du Blanc, ses stratégies, dissimulations ou trahisons, l'effort aussi pour comprendre le contexte élargi et international. Car la guerre de 1917 se joue dans les plis de la Grande Guerre, celle de 1914-1918, et le Kaiser Guillaume n'est pas un inconnu pour les Kanaks dont certains espèrent qu'il enverra des fusils et parviendra à battre les Français. Adrian Muckle livre au public français une synthèse de sa thèse en une centaine de pages très circonstanciées sur le déroulement des faits, les causes immédiates et profondes et les conséquences proches ou plus lointaines. Cette description historique essentiellement appuyée sur l'analyse des archives est le point de départ des 600 pages qui suivent, fondées sur l'exploration minutieuse de 17 récits kanaks contemporains ou postérieurs à l'événement; points d'appui d'une explicitation anthropologique et historique de la guerre de 1917, de sa mémoire et transmission dans les univers kanaks impliqués. La guerre, enfin, est aussi au cœur du travail de Michel Naepels non plus comme événement à décrypter mais comme ligne d'analyse fondamentale inspirée des propos de Michel Foucault cités en introduction : «La guerre peut-elle valoir analyseur des rapports de pouvoir?»(cité in Naepels, p. 11). Elle constitue le «fil conducteur» d'une enquête consacrée exclusivement à la région de Houaïlou (côte est de la Grande Terre) saisie sur un temps long de l'histoire qui court de 1847, date de l'arrivée des premiers Européens, jusqu'aux années 2000. La guerre, grande ou petite, synonyme du conflit, important ou banal, sert de levier heuristique pour comprendre la violence, ses conventions d'usage et ses modalités d'exercice ou de contrôle ainsi que les rapports sociaux ainsi révélés, et ce, à travers l'analyse détaillée de situations sociales singulières et précisément décrites, choisies « en coupe » dans l'histoire coloniale et postcoloniale de Houaïlou sur l'étendue de la période considérée. La guerre est ici conjurée en tenant compte de la polysémie du terme, contrôlée ou déployée, au grand jour ou en secret. Elle est au cœur de l'ouvrage ; les pratiques guerrières, la nature et la forme des violences, les forces et les collectifs mobilisés, les négociations, évitements, résolutions des conflits ou au contraire leur persistance et résurgence. L'histoire de Houaïlou est saisie par la guerre, celle qui est menée par les Kanaks et par les Européens au cœur même du processus de colonisation. 


\section{Les enjeux de la fabrique narrative ou les multiples voix d'un livre : la guerre de 17 vue}

de Koné

Les sanglots de l'aigle pêcheur est un livre dense de 713 pages bâti en trois parties dont la première est signée d'Adrian Muckle tandis que les deux autres sont cosignées par les trois contributeurs. Disons que la première partie de l'ouvrage est clairement identifiée sous le nom de l'historien pour introduire historiquement les évènements qui ont eu lieu dans la région nord de la Nouvelle-Calédonie en 1917. Les deux suivantes intitulées «La mémoire kanak de la guerre de 1917 » et «Poétique politique kanak» qui constituent l'essentiel de l'ouvrage sont plus composites. La figure d'Alban Bensa, son expérience de recherche en Nouvelle-Calédonie commencée en 1973 et poursuivie jusqu'à aujourd'hui, son rôle dans le choix des récits kanaks insérés, certains directement issus de ses enquêtes de terrains ainsi que l'analyse qu'il en fait, s'impose à l'évidence comme largement prédominante. Il s'agit, on le sent, de deux parties rédigées par sa main (hormis les récits présentés) et qui veulent restituer l'ampleur de son effort de recherche accompli depuis plus de 30 sur le terrain calédonien. Adrian Muckle a bien sûr apporté certains éclairages et Yvon Goromoedo a indéniablement contribué à la traduction de textes récents recueillis en langue paicî et à leur interprétation. Mais il y a un ouvrage dans l'ouvrage, de la plume d'Alban Bensa.

Ces deux parties, cependant, font entendre d'autres voix. Alban Bensa mentionne et remercie à différentes reprises son collègue Jean-Claude Rivierre qui l'accueillit sur le terrain en 1973, linguiste déjà reconnu et élève d'André-Georges Haudricourt. Celui-ci travailla d'abord, à partir de 1965, dans le centre et le nord calédonien avec sa femme, Françoise Ozanne Rivierre, linguiste distinguée elle aussi, aujourd'hui décédée. Ces deux linguistes du CNRS ont enrichi et renouvelé la linguistique néo-calédonienne. On leur doit aussi un travail approfondi de recueil de textes dans plusieurs langues kanak ; certains récits soigneusement traduits et annotés sont repris dans l'ouvrage. Ainsi le texte de Téâ Alphonse Goa recueilli en langue fwâi par Françoise Ozanne Rivierre en1976. Ou encore deux longues poésies transcrites par Jean-Claude Rivierre et reproduites dans la troisième partie de l'ouvrage : l'une enregistrée en 1973 auprès de Cau Pwënyi Mêrêatü, et l'autre qu'Alban Bensa qualifie de texte majeur de la littérature kanak du XXe siècle, composée en paicî par deux hommes de la région de Poindimié puis transmise à un jeune pasteur 
(Cau Eleisha Näbai) travaillant pour Maurice Leenhardt et récupérée par André Georges Haudricourt, pour être finalement travaillée par Jean Claude Rivierre et Dui Novis Pöömô. La contribution de Jean-Claude Rivierre dans la fabrication de l'ouvrage reflète l'intensité des collaborations qu'ont eu l'anthropologue et le linguiste sur le terrain dans les années 1970-1990, au point que nous n'aurions pas été étonnés de voir apparaitre son nom parmi les co-auteurs.

La force de l'ouvrage est aussi de rendre hommage à la qualité littéraire et à la mémoire extraordinaires des érudits kanak qui, depuis 1919 et jusqu'à aujourd'hui, ont voulu fixer, en vers ou en prose, les évènements de 1917 et leurs interprétations vécus par les acteurs eux-mêmes ou transmis à leurs descendants. On a cité plusieurs noms et on pourrait les citer tous car derrière chaque récit, des plus anciens aux plus récents, il y a une voix, une personne, une expérience, une histoire, évoquant des lieux précis, des alliés, des clans, des combats, des trahisons et des soutiens, des itinéraires, des fuites La dynamique mémorielle en Nouvelle Calédonie est extrêmement féconde aujourd'hui comme en témoigne la cérémonie décrite par Alban Bensa qui s'est tenue en 2009 à Cémû autour d'une stèle installée à la mémoire de Bwëé Noël Tiamou, chef de guerre kanak, tué en 1918 par un bagnard, originaire du Maroc et condamné par la Cour d'assise d'Oran en 1889. L'intense émotion produite dans la région par cette commémoration parmi les familles qui furent impliquées d'un bord ou de l'autre dans les affrontements a poussé une femme, Anaïs Nägèè, descendante des parents maternels de Bwëë Noël Pwatiba qui ne se sont jamais exprimés publiquement jusqu'alors, à apporter son témoignage. Elle a ainsi enfin osé prendre la parole pour contribuer à honorer son parent et évoquer le rôle protecteur des maternels. Cette dynamique mémorielle très vive et que l'on voit aussi à l'œuvre avec « le retour de la tête d'Ataï » et le souvenir de la grande insurrection (guerre) de 1878, remonte finalement assez loin. Non sans malice, Alban Bensa rappelle que ceux qu'on nomme habituellement « les élèves » de Maurice Leenhardt furent aussi d'excellents professeurs pour celui qui fut « leur meilleur élève européen », (A. Bensa, Y. Goromoedo, A. Muckle, p. 436) avide d'apprendre la langue (et très fin connaisseur de l'Ajië, langue de Houaïlou), les pratiques, les croyances, les parentés et autres. Il incita ses «élèves » à écrire et ceux-ci répondirent avec passion pour fixer, transcrire, témoigner, raconter comme nous donne à le voir les récits datant de 1919 ici publiés. Cette dynamique écrite et orale n'a cessé de se poursuivre dans les décennies qui ont suivi.

Pour autant la lecture de ces textes kanak n'est pas simple pour le profane et les éclairages d'Alban Bensa sont très utiles pour expliciter les logiques évoquées, préciser l'ordre des parentés, 
expliciter les conflits de hiérarchies et d'autorité, les distinctions entre «vrais chefs » et les autres, éclairer l'impact de l'organisation coloniale des chefferies administratives, des recrutements forcés, de la mise place de l'impôt de capitation, l'impact aussi de l'évangélisation, «ces entrepreneurs en christianisme » que sont pour Alban Bensa les missionnaires qui voient dans la guerre de 1917 et surtout dans la répression qui s'ensuit, une aubaine pour faire progresser leur cause et rallier les vaincus à leur église.

Adrian Muckle dans les trois chapitres de l'ouvrage nous livre une analyse historique très bien organisée, partant d'une description précise de l'affrontement premier qui ouvrit les hostilités, le 28 avril 1917, lors d'une cérémonie supposément réconciliatrice entre les hommes de Cémü, Pana et Pwënäki avec « les policiers (kanak) » et le petit chef de la réserve KoniamboGrombao. Le contexte est très tendu puisque ces derniers usent de la force pour recruter des «volontaires » kanak pour partir au front en France. Il s'agit d'une deuxième vague de recrutement, la première ayant eu lieu en 1916 suite à l'appel lancé, par la France, à la fin de l'année 1915, aux « indigènes » de l'Empire non mobilisables de venir renforcer « de leur plein grés » les rangs de l'armée. Le « volontariat » prend un goût amer dans le nord calédonien où on voit s'exercer toutes sortes de pressions sur les «grands chefs » et les «petits chefs» pour que les hommes s'engagent. Le zèle du « petit chef » de Koniambo crée des tensions avec Noël et ses guerriers que le chef des affaires indigènes prétend calmer par une cérémonie de réconciliation qui est en fait, selon toute vraisemblance, un piège tendu aux récalcitrants. Ces derniers, avertis, loin de se soumettre, insultent du haut des collines les soldats et narguent les balles jusqu'à la scène restée célèbre dans la mémoire kanak du frère de Noël échappant miraculeusement à la mitraille (grâce dit on à la protection des ancêtres) et montrant son cul à la troupe. La guerre est déclarée et enflamme rapidement le haut de Koné sur la côte ouest mais se déplace au cours de l'année sur la côte est, dans la vallée de la Tipidjé puis vers Hienghène. On ne reviendra pas ici sur le détail des affrontements si ce n'est pour dire qu'ils remontent à rebours les chemins coutumiers suivie par la monnaie de guerre qui a été lancée, semble-t-il, sur la côte est par le grand chef de Hienghène auprès des clans qui lui sont apparentés et dévoués. Les raisons de la guerre sont multiples et s'inscrivent dans l'épaisseur d'un passé immédiat ou plus lointain auquel les Kanaks se réfèrent pour agir ou non, s'engager auprès « des rebelles », rester neutres ou au contraire partir avec les colonnes françaises comme auxiliaires. Le souvenir des guerres passées entre Kanaks, l'expérience des humiliations et répressions exercées par les forces armées de la 
colonisation, les spoliations des terres aux profits des colons, la pression du bétail, les interdictions de circulation et les obligations de travail liées à l'application du régime de l'indigénat depuis 1887, le refus ou l'acceptation de la conversion sont autant d'éléments qui expliquent, en fonction de circonstances très localisées, les motivations à l'aune desquelles se forgent les décisions. Les récits kanak qui nous sont donnés à lire et que l'on peut entendre en langue grâce au CD qui vient magnifiquement compléter l'ouvrage, racontent encore et encore, les stratégies d'alliance, les engagements, les retournements, les doutes et les trahisons dans un contexte de violence exacerbée par une guerre sans pitié, menée par des colonnes militaires françaises pratiquant, ici comme dans d'autres colonies, une politique systématique de terre brûlée tout en encourageant les exactions parmi les hommes, soldats ou auxiliaires.

\section{Houaïlou à l'épreuve de la colonisation ou la longue durée des guerres et des}

\section{alliances.}

C'est aussi le théâtre de la guerre qui ouvre le livre de Michel Naepels dans un premier chapitre consacré à la région que l'on nommera finalement Houaïlou entre 1847 et 1881 . Le récit que l'auteur nous livre de ces premiers contacts avec l'Europe - l'activité des chercheurs de santal, l'arrivée d'émigrants en provenance de San Francisco sur le chemin de l'or, le sort funeste qui les attend dans les vallées de l'arrière pays, le débarquement des troupes guidées de fait par des guerriers venus de Canala et, enfin, le déchainement de la violence qui mêle la fureur des armes à des scènes d'anthropophagie, est absolument saisissant. Outre la qualité des descriptions choisies, on notera l'extrême concision du propos et l'attention aigüe portée aux «traces » très incomplètes que livrent les archives européennes inhabiles à décrire précisément les univers sociaux alors à peine entrevus. Les faits sont trop anciens et extérieurs aux logiques sociales pour que les souvenirs kanaks les aient fixés. Michel Naepels lance son enquête à partir de cet événement premier, la disparition d'un groupe de chercheurs d'or, signalé semble-t-il par un kanak de Canala qui oriente la répression vers la tribu d'Aliki Wangâ, chefferie du bord de mer, pour des raisons proprement kanak liées à des rivalités plus anciennes. On notera que Michel Naepels, à aucun moment, ne cherche à reconstituer des ordres sociaux pré-coloniaux comme des donnés et le mot chef ou chefferie sont à ce stade utilisés avec une extrême prudence, renvoyant à des personnages ou des familles suffisamment éminents pour être repérés comme tels par les 
autorités coloniales. Partant de ces noms qui apparaissent dans les sources et sous la visée des canons Aliki Wanga, Aliki Ykà , Michel Naepels tente l'identification et s'engage dans l'histoire prosopographique d'individus ou familles qui vont finalement rallier l'autorité française du fait de cette terreur première que celle-ci leur inflige. L'alliance est solide car, par leur descendance, ces familles vont fournir les principaux «chefs administratifs» de Houaïlou au cours des cinquante années qui suivent, consolidés sous le titre de «grand chef » (grand chef Mèjâ) et « petit chef » à partir de 1897. Les guerriers Houaïlou, on le sait, seront par la suite de fidèles auxiliaires engagés en nombre dans les répressions coloniales sur la Grande Terre mais aussi sur les théâtres extérieurs au cours des deux guerres mondiales.

C'est en suivant le fil de la guerre et en particulier l'un des objets essentiels de l'arsenal guerrier et magique kanak, les «pierres propitiatoires» que Michel Naepels passe d'un premier tableau centré sur la période de ralliement aux autorités françaises des chefferies du littoral entre 1847-1881 à un second tableau traitant de façon complexe l'histoire des relations entre ces chefferies du littoral s'appuyant sur leur alliance avec les autorités françaises pour solder des conflits anciens remontant au XVIIIe siècle via des opérations de répression menées en 1867 puis via le cantonnement organisé à la fin du XIXe siècle. C'est par une enquête minutieuse croisant les entretiens approfondis qu'il mène avec «les vieux », la lecture qu'il fait d'un récit livré par l'un des plus fins informateurs de Maurice Leenhardt (Bwêêyöuu Ërijiyi, apparenté à la famille du grand chef Mèèjâ Néjâ, fin connaisseur de sa société et converti protestant), les sources dont il dispose concernant le passage des collecteurs et des ethnographes dans la région avant la guerre de 14-18 et l'analyse critique qu'il mène de l'œuvre ethnologique de Leenhardt que Michel Naepels parvient à éclairer finement l'agency des acteurs kanaks. Sont éclairées, en particulier, les stratégies de la famille Néjâ et des ses alliés visant à l'accaparement du pouvoir local et le refoulement de certains clans en appui à la politique de cantonnement imposée par le gouverneur Feillet entre 1897 et 1903. De même est analysé le rôle complexe d'un converti tel que Bwêêyöuu à qui l'on doit une contribution remarquable à la connaissance anthropologique, via la transmission à Leenhardt de son savoir extraordinairement précis sur les pratiques et croyances dans Anciens. Mais Bwêêyöu transmet pour mieux abandonner, des croyances qu'il récuse et dénonce, pour lui et les siens, au nom de son adhésion à la «nouvelle religion» - le protestantisme - dont il devient un agent prosélyte actif encourageant le don ou l'abandon des objets comme les pierres de guerre ou autre au risque de les voir définitivement disparaître. 
Le troisième tableau que propose Michel Naepels couvre la période 1897-1939 marquée par le triomphe de l'ordre colonial appuyé sur un service de l'indigénat tout puissant relayé par les gendarmes, les «grands chefs», les «petits chefs» pour mieux servir la société et l'économie coloniales. Mèèjâ Néâ, nommé grand chef du district de Houaïlou en 1897 illustre la complexité d'une position essentielle dans la nouvelle gouvernementalité, à la fois homme de main du pouvoir, agent actif pour libérer les terres qui doivent être données aux colons, appliquant avec zèle les règlements qui lui donnent le droit de punir ses sujets, d'imposer les réquisitions, de recruter pour les colons, d'interdire les déplacements, fêtes, rencontres etc. Mais Mèèjâ Néâ s'enfonce aussi dans l'alcool comme tant d'autres et refuse visiblement de se soumettre à l'arrogance de colons et commerçants du lieu. Il est exilé à Maré (Iles Loyauté) où il embrasse le protestantisme entre 1899 et 1902 puis subit l'humiliation, en 1912, de voir son district divisé en quatre et son pouvoir diminué par la nomination de trois autres «grands chefs » issues d'autres familles. La devise « diviser pour mieux régner » pratiquée par les Français est ici illustrée magistralement afin de mieux manipuler les «grands chefs » et les impliquer dans le contrôle colonial jusque dans l'application de mesures sanitaires autoritaires et ségrégatives contre la lèpre ou la peste. En dépit de la dénonciation récurrente des autorités françaises du « despotisme des chefs » et leur volonté affichée de limiter les pouvoirs de ces derniers par la création « de conseils des anciens » en 1919 (qui restèrent fictifs jusqu'aux années 1940), ceux ci restent les maillons essentiels «de l'ordre ségrégé et policier qui s'est progressivement mis en place au cours de la seconde moitié du XIXe siècle» qui constitue, ajoute Michel Naepels «le modèle de référence implicite et paradoxal quand est évoquée «la coutume » aujourd'hui en Nouvelle-Calédonie » (M. Naepels, p. 107). Cette remarque glissée incidemment est d'une importance cruciale puisqu'elle engage une réflexion sur les effets d'héritage de la construction coloniale dans la période contemporaine post coloniale.

Le « post colonial » renvoie ici à la rupture fondamentale que constitue, dans le contexte de la Nouvelle-Calédonie, l'abolition du régime de l'indigénat en 1946 et la suppression d'un ensemble d'interdits ou d'obligations qui, de facto, réduisent considérablement les pouvoirs acquis sous la période antérieure par les «grands» et «petits» chefs. A cette « révolution» s'ajoute l'élargissement de la citoyenneté et l'accès progressif de tous, hommes et femmes, Kanak et non Kanak, au suffrage universel en 1957. L'entrée en politique signe l'émergence de nouveaux partis politiques et de nouvelles élites élues. 
Les trois tableaux suivants que nous propose Michel Naepels traitent des contrecoups de ces transformations profondes à Houaïlou entre 1945 et 2012 sur les nouvelles formes de gouvernementalités locales et de mobilisations en évoquant trois moments et une série d'évènements. Au cours des années 1950, Houaïlou est profondément marqué par une série d'affrontements : sur le plan économique, la montée de coopératives agricoles concurrentes, sur le plan religieux, des accusations brutales menés par des Kanaks protestants contre des «païens » usant de «pouvoirs anciens et «maléfiques » auxquels s'ajoutent les déchirements de la mission de Do Néva à l'origine d'un mouvement éducatif dissident et de tensions très vives et enfin, sur le plan politique, de profondes divisions au sein l'Union Calédonienne, le grand parti de l'aprèsguerre, dont l' impact local est très fort. Sont explorés ensuite les événements de 1984-1998 vus de Houaïlou, un fait divers en 1987 conduisant à la mort d'un homme dans le contexte de la réforme foncière et la redistribution des terres spoliées par les colons aux Kanaks, et une scène de «violence ordinaire » en 1991 à l'intérieur d'une même famille liée aussi à des questions de terre. Enfin, le dernier tableau présente des « scènes éparses » de la vie quotidienne à Houaïlou dans les années 2000 qui font suite à la signature de l'accord de Nouméa en 1999. La reconfiguration d'une gouvernementalité postcoloniale se traduit, semble-t-il, par un fort investissement dans les cérémonies collectives marquant les deuils et levées de deuil, les naissances et les mariages, occasions maintes fois renouvelées pour travailler la pacification de l'espace villageois et le consensus. Mais le contexte pourtant tire plutôt dans le sens d'un éclatement et de la mise à l'épreuve des solidarités villageoises : l'insertion dans l'économie salariale, les mobilités hors de Houaillou vers Nouméa, l'agrandissement des réserves et de terres coutumières. S'ajoutent à cela de nouveaux mots d'ordre ou de nouvelles dynamiques, le « destin commun » prôné par l'accord de Nouméa, la création de formations politiques ( la FCCI par exemple) ou d'institutions coutumières nouvelles comme le Sénat (coutumier) ou encore la forte attractivité des grands chantier miniers du nord etc.., autant d'éléments mettant terriblement à mal l'ancienne organisation locale issue de la période coloniale et centrée sur la tribu, la réserve, le petit chef, le district et le grand chef.

Si Michel Naepels voit dans la période contemporaine, post événements «la pacification des rapports sociaux, particulièrement sensible dans les espaces du commun : les cérémonies, les villages, les tribus et les lieux de corésidence [...]» (M. Naepels, p. 215), il insiste, pourtant aussi, sur la persistance et la récurrence des conflits locaux et des violences intrafamiliales ou 
non, liés souvent à des hostilités fonciers anciens et aggravés par l'alcoolisation et le cannabis dont abuse une jeunesse désœuvrée dont beaucoup s'inquiètent.

Nous laissons au lecteur le soin de découvrir à la lecture les chemins que Michel Naepels choisit d'emprunter pour tenter de décrypter la période post coloniale. Notons seulement que la ligne de cohérence se perd quelque peu au fil du texte à travers une multitude de formes de mobilisation collectives ou stratégies individuelles que l'auteur cherche à relier sous le couvert de la conflictualité et du principe de guerre rejoué sous de multiples formes nouvelles. Mais le lecteur peine à tenir le fil à mesure que les descriptions d'évènements disjoints se diffractent et que l'analyse, faisant preuve d'une grande prudence, se refuse à l'explication causale et choisit d'anonymiser les entretiens pour ne pas mettre en danger les interlocuteurs laissant une impression d'incomplétude et d'inachevé que Michel Naepels revendiquera peut être. L'ambition de couvrir une si longue période (1847-2012), quoique rigoureusement rapportée à la localité de Houaiilou et plus précisément au monde kanak qui l'habite, exigeait un effort dont on mesure l'importance mais présentait le risque d'une certaine dilution de l'intelligibilité des situations contemporaines.

Reste l'apport de ces ouvrages que nous venons de présenter dont l'immense mérite de poser les jalons d'une histoire «épaisse » qui rend justice aux dynamiques des acteurs kanaks, à leurs logiques d'action et leur subjectivité. S'ouvrent ainsi de multiples pistes de réflexion et de recherche dont historiens, anthropologues ou politistes gagneront à s'emparer pour penser d'une façon complexifiée, le passé et le présent de ce pays calédonien.

Isabelle MERLE

CNRS-CREDO, Aix Marseille Université/ EHESS

Adresse postale?

Courriel? 